\title{
Sound comparison of seven TMS coils at matched stimulation strength
}

Lari M. Koponen ${ }^{a}$, Stefan M. Goetz ${ }^{a, b, c}$, Debara L. Tucci ${ }^{d}$, Angel V. Peterchev ${ }^{a, b, c, e}$

a Department of Psychiatry and Behavioral Sciences, Duke University, Durham, NC 27710, USA

${ }^{b}$ Department of Electrical and Computer Engineering, Duke University, Durham, NC 27708, USA

c Department of Neurosurgery, Duke University, Durham, NC 27710, USA

d Department of Surgery, Duke University, Durham, NC 27710, USA

e Department of Biomedical Engineering, Duke University, Durham, NC 27708, USA

\section{Correspondence}

Angel V. Peterchev

40 Duke Medicine Circle, Box 3620 DUMC, Durham, NC 27710, USA

angel.peterchev@duke.edu

919-684-0383

\section{Abstract}

Background: Accurate data on the sound emitted by transcranial magnetic stimulation (TMS) coils is lacking.

Methods: We recorded the sound waveforms of seven coils with high bandwidth. We estimated the neural stimulation strength by measuring the induced electric field and applying a strength-duration model to account for different waveforms.

Results: Across coils, at maximum stimulator output and $25 \mathrm{~cm}$ distance, the sound pressure level (SPL) was 98-125 dB(Z) per pulse and 75-97 dB(A) for a $15 \mathrm{~Hz}$ pulse train. At $5 \mathrm{~cm}$ distance, these values were estimated to increase to $112-139 \mathrm{~dB}(\mathrm{Z})$ and $89-111 \mathrm{~dB}(\mathrm{~A})$, respectively.

Conclusions: The coils' sound was below, but near, relevant exposure limits for operators and may exceed some limits for the subject. Exposure standards may inadequately capture some risks to hearing. For persons near operating TMS coils we recommend hearing protection, and we consider it essential for the TMS subject.

\section{Highlights}

1. Coil click varies by over $20 \mathrm{~dB}(\mathrm{Z})$ between TMS coils at matched stimulation strength.

2. Close to TMS coil, sound pressure level may reach nearly $140 \mathrm{~dB}(\mathrm{Z})$.

3. For rTMS, the continuous sound level can exceed $110 \mathrm{~dB}(\mathrm{~A})$.

4. Hearing protection is recommended during TMS, especially for the subject.

\section{Keywords}

Transcranial magnetic stimulation, coil click, sound, stimulation strength 


\section{Introduction}

Transcranial magnetic stimulation (TMS) activates cortical neurons by electromagnetically inducing an electric field (E-field) pulse with a coil placed on the subject's scalp. In addition to the desired E-field stimulation, the pulse causes mechanical vibrations in the coil, producing a brief but very loud sound impulse-often referred to as TMS coil click [1-2].

The sound pulse can potentially cause hearing loss. This risk can be mitigated with adequate hearing protection [3-5]. However, should the hearing protection fail, TMS can cause permanent hearing loss [6]. The sound also evokes unwanted neural activation that deteriorates the specificity of TMS and confounds functional brain imaging data [7], which can be only partially mitigated with noise masking [8].

Due to these side effects, the TMS safety consensus group suggested in 2009 that "the acoustic output of newly developed coils should be evaluated and hearing safety studies should be conducted as indicated by these measures" [9]. Since then, specific stimulator-coil combinations have been evaluated with hearing safety measurements [4-5], and a comparison between coils has been published [10]. To address methodological limitations of the latter, namely sound level measurements intended only for continuous (i.e., not impulsive) sounds based on IEC 61672 [2], and to expand the range of compared devices, we conducted measurements of the coil click in a total of seven coils for three stimulators and normalized the stimulation strength across the various configurations. Moreover, we interpret the results for single pulses as well as for repetitive TMS (rTMS) in the context of various standards for sound exposure limits.

\section{Material and methods}

\section{TMS devices}

We tested three stimulators with a total of seven different figure-of-eight coils. The model information of the tested stimulators and coils is provided in Table 1. The "stimulator output" refers to the stimulator capacitor voltage, expressed as a percentage of its maximum (maximum stimulator output, MSO), which determines the TMS pulse amplitude.

\section{Sound recording}

A microphone was pointed at the center of the head-side of each coil at a distance of $25 \mathrm{~cm}$ (see Supplementary Fig. S1B). We sampled 10 pulses per stimulator output at $10 \%$ to $100 \%$ of MSO in $10 \%$-MSO steps. The pulses at each stimulator output were separated by an interstimulus interval of 2-3 s. To isolate the coil click from sound originating from the stimulator, the coils were placed inside a soundproof chamber (Model 402.a; Industrial Acoustic Company, USA). To suppress echoes in the recordings, the relevant chamber interior walls were covered with $50 \mathrm{~mm}$ thick open-cell foam pads. To convert the digital signal into pascals, the system was calibrated with a $1 \mathrm{kHz}, 1$ Pa reference sound pressure source (Extech Instruments 407722, Extech, USA). To allow accurate measurement, we used an omnidirectional pressure microphone (Earthworks M50, Earthworks Audio, USA), which has a flat frequency response ( -1.1 to $+0.2 \mathrm{~dB}$ referenced to our $1 \mathrm{kHz}$ calibration tone) from $3 \mathrm{~Hz}$ to $50 \mathrm{kHz}$ and is free of distortion for sound pressure level (SPL) up to $140 \mathrm{~dB}(\mathrm{Z})$. The microphone was connected to a high-input-level-capacity preamplifier (RNP8380, FMR Audio, USA) and an audio interface with a sample rate of $192 \mathrm{kHz}$ (Behringer U-Phoria UMC404HD, Behringer, Germany).

\section{Sound processing}

In processing the raw audio, the first pulse at each stimulator output was excluded as an outlier, since it deviated from the subsequent pulses for all devices especially at low stimulator outputs. The signal was low- 
pass filtered at $60 \mathrm{kHz}$ to remove high-frequency readout noise, and high-pass filtered at $60 \mathrm{~Hz}$ to remove lowfrequency sounds which mostly originated from the air conditioning. The cut-off frequency for the latter filter was selected post-hoc, as none of the coils produced sound that deviated from the background noise at any frequency below $100 \mathrm{~Hz}$. The high-and low-pass filters were fourth-order Butterworth type applied in both forward and reverse time, resulting in flat frequency response from $80 \mathrm{~Hz}$ to $50 \mathrm{kHz}$ (within $1 \mathrm{~dB}$ ). For these data, we computed the peak SPL [11] in a $0.2 \mathrm{~s}$ window around the pulse, the duration of the sound impulse defined as the period for which the sound pressure was within $20 \mathrm{~dB}$ of its peak value, and 1/3-octave spectra with the poctave function (Matlab R2018a, Mathworks, USA).

In addition, to estimate the continuous sound level due to rTMS, we synthesized 10 seconds of rTMS sound as a superposition of individual pulses repeated at $15 \mathrm{~Hz}$. This frequency was chosen as the highest average frequency that is FDA-cleared for clinical use with the figure-of-eight type coils that we characterized, since the $15 \mathrm{~Hz}$ rTMS trains are equally loud to theta burst stimulation comprising bursts of three pulses at $50 \mathrm{~Hz}$, repeated at $5 \mathrm{~Hz}[12]$.

\section{Estimation of stimulation strength}

To compare the sound between different coils, which provided different stimulation waveforms and MSO, we normalized their stimulation strengths as follows. First, we measured the induced electric field (E-field) corresponding to a depth of $15 \mathrm{~mm}$ under the center of the coil in an $85 \mathrm{~mm}$ sphere approximating the head [13] using a 70-millimeter-high triangle-loop probe [14-15]. The E-field was sampled at $100 \mathrm{MHz}$ with an oscilloscope (Tektronix MDO3054, Tektronix, USA). To obtain the effective stimulation strength, the E-field waveform was digitally low-pass filtered with a time constant of $200 \mu \mathrm{s}$, corresponding to the approximate strength-duration time constant in primary motor cortex [16-18]. The stimulation strength, defined as the absolute peak of the filtered waveform, was then scaled relative to the average resting motor threshold (RMT) by denoting $100 \%$ RMT to correspond to $50.3 \%$ MSO for the Magstim 70mm Double Coil driven by a Magstim Rapid biphasic stimulator [19]. Based on the variance of RMT across individuals, for about $95 \%$ of subjects, the individual RMT should lie between $75-125 \%$ of this value [19].

\section{Sound spectrum weighting and exposure limits}

Choosing an appropriate exposure limit for TMS sound is not straightforward. In addition to the common limit of $115 \mathrm{~dB}(\mathrm{~A})$ time-averaged steady-state sound level for short daily exposure [20-23], we must also consider the impulsive nature of the sound, which is similar to a small explosion, e.g., shooting a firearm, in terms of duration and peak sound pressure [24]. Consequently, measurement protocols for such sounds should provide good reference for the measurement protocol of TMS sound. Several safety standards give an instantaneous SPL limit of $140 \mathrm{~dB}$, but with either A-weighting [22], C-weighting [23], or Z-weighting [20-21,25] (for frequency-weighting specifications, see Supplementary Fig. S2). A- and C-weighing only limit the sound in the hearing range (as they are defined only up to $20 \mathrm{kHz}$ ). With these, the limits for potential near-ultrasound content has to be considered separately. However, the relevant standards do not contain such separate impulsive limits, but only limits for continuous sounds of various durations, varying between $75-110 \mathrm{~dB}$ at $20 \mathrm{kHz}$ and 110-115 dB at $25 \mathrm{kHz}$ or above [26]. To avoid the need for two separate metrics, we use the Zweighted $140 \mathrm{~dB}$ peak SPL from MIL STD 1474E [25], which also imposes a constraint on the impulsive nearultrasound content. We also considered the sound level of rTMS pulse trains using A-weighting (slow time weighting) per IEC 61672 [11] computed with splMeter from the Audio Toolbox (Matlab R2018a, Mathworks, USA). 


\section{Results}

\section{Coil electric field and stimulation strength}

The peak E-field at 100\% MSO varied between 140-260 V/m across the tested coils, and it had nearly linear relationship to the stimulator output setting for all coils (Fig. 1A). The pulse durations varied between 176$353 \mu$ s and were largely stable across the output range of the devices except for the NeuroStar iron-core coil, which had about $19 \%$ longer pulse at $10 \%$ MSO than at $100 \%$ MSO (Fig. 1B). Consequently, for all but the ironcore coil, the devices showed an approximately linear relationship between the simulator capacitor voltage and the estimated stimulation strength (Fig. 1C) (NeuroStar devices, however, have an output calibration feature that can compensate for this nonlinearity).

\section{Coil sound characteristics}

At a distance of $25 \mathrm{~cm}$, the seven coils had a wide range of peak SPL, 98-125 dB(Z) at 100\% MSO and 92$114 \mathrm{~dB}(\mathrm{Z})$ at the estimated $120 \% \mathrm{RMT}$, with the MagVenture MRi-B91 being the quietest and the MagVenture C-B60 being the loudest (Fig. 1D). There were three distinct click types: very brief and sharp (1-2 ms) from conventional MagVenture coils; medium length (6-7 ms) from NeuroStar coil and Magstim 70mm Double Coil, and long (>10 ms) from the MagVenture MRi-B91 coil and Magstim AirFilm Coil which have double casing (Fig. 1E). For most coils, the sound duration was comparable across stimulation strengths. For Magstim AirFilm Coil, the click duration decreased from $35 \mathrm{~ms}$ to $9 \mathrm{~ms}$ when the stimulator output was increased from $20 \%$ MSO to $100 \%$ MSO. All coils produced broad sound spectra with peak varying between $1-8 \mathrm{kHz}$ and spectrum extending up to $30 \mathrm{kHz}$ (Fig. 2). At 100\% MSO, the 1/3-octave band had sound level between 40-69 dB at $20 \mathrm{kHz}$ and $41-64 \mathrm{~dB}$ at $25 \mathrm{kHz}$. Peak SPL varied approximately proportionally to the stimulation strength squared (Fig. 1D). For example, increasing the stimulation strength by $20 \%$ increases the sound level by approximately $3 \mathrm{~dB}$. This is expected from first principles, since the mechanical force causing the sound is a product of the magnetic field and coil current, both of which are proportional to the stimulator capacitor voltage.

The continuous sound for simulated $15 \mathrm{~Hz}$ rTMS at 120\% RMT ranged 70-90 dB(A), with NeuroStar being the loudest and MagVenture MRi-B91 remaining the quietest but with a much smaller margin than for peak SPL (Fig. 1F). Doubling the repetition rate increases the sound level by approximately $3 \mathrm{~dB}$; this scaling is valid for frequencies up to about $100 \mathrm{~Hz}$, where the sound levels are $8 \mathrm{~dB}$ higher than the values shown in Fig. $1 \mathrm{~F}$. Table 2 summarizes how the data presented in Fig. 1 can be scaled approximately to various stimulation strengths, rTMS train frequencies, and coil distances.

\section{Discussion}

Our measurement setup mostly followed the suggested measurement procedures in MIL STD 1474E [25], which considers impulse sounds, such as from explosions, which are similar in their characteristics to the coil click. We measured the far-field sound, at a distance larger than the characteristic dimensions of the TMS coil. Our microphone was placed $25 \mathrm{~cm}$ away from the center of the coil, compared to coil-ear distances as small as $5 \mathrm{~cm}$ for the TMS subject. We chose the $25 \mathrm{~cm}$ distance to avoid either (1) having to sample the spatial distribution of the sound or (2) underestimating the SPL due to measuring near a nodal point of a standing wave pattern in the near field $[2,18]$. The sound pressure from TMS coils decays approximately inversely to the distance down to $5 \mathrm{~cm}$ [27] (see Table 2); therefore, the sound pressure at $5 \mathrm{~cm}$ from a coil is about $14 \mathrm{~dB}$ higher than the sound pressure measured at $25 \mathrm{~cm}$. Thus, the peak SPL of the seven coils can be estimated to range from 106 to $129 \mathrm{~dB}(\mathrm{Z})$ at $120 \% \mathrm{RMT}$ and $5 \mathrm{~cm}$ from the coils. The $22 \mathrm{~dB}$ difference between the coils is 
preserved with this scaling; this is comparable to the typical attenuation of air-conducted sound obtained with simple hearing protection [22]. Considering the extreme cases we can estimate, single pulses at $100 \%$ MSO and $5 \mathrm{~cm}$ from the coils, which for some TMS targets is comparable with the distance to the subject's ear, produce SPL of $112-139 \mathrm{~dB}(\mathrm{Z})$.

As with the sound of single TMS pulses, there were large differences in the time-averaged steady-state sound levels of pulse trains for rTMS. Extrapolating to a $5 \mathrm{~cm}$ distance, a $15 \mathrm{~Hz}$ rTMS train has a sound level of 84$104 \mathrm{~dB}(\mathrm{~A})$ at $120 \% \mathrm{RMT}$. At 100\% MSO, the sound level would reach 89-111 dB(A). These values would be approximately $2 \mathrm{~dB}$ lower for $10 \mathrm{~Hz}$ rTMS trains. The OSHA limit for continuous sound level is $115 \mathrm{~dB}(\mathrm{~A})$ for short durations ( $<15 \mathrm{~min}$ ) and degrades by $5 \mathrm{~dB}$ per doubling of exposure duration to $90 \mathrm{~dB}(\mathrm{~A})$ for exposure of 8 hours [20-21]. Even in case of a TMS operator administering multiple rTMS sessions per day and remaining in close proximity of the coil at $25 \mathrm{~cm}$, our estimated sound levels are below the OSHA limit for occupational exposure (with the possible exception of very high repetition rates, e.g. $100 \mathrm{~Hz}$ used in magnetic seizure therapy). However, these exposure limits are approached for TMS subjects. Moreover, for most coils the sound level extrapolated to a distance of $5 \mathrm{~cm}$ can exceed the lowest near-ultrasound limit of $75 \mathrm{~dB}$ at $20 \mathrm{kHz}$ [26]. Therefore, the use of hearing protection for the TMS subject is advisable.

In this dataset of commercial stimulators and coils, all coils had most of their sound in the hearing range. All coils, however, also had considerable sound level in the $20-30 \mathrm{kHz}$ range, with $15 \mathrm{~Hz}$ rTMS at $100 \%$ MSO exceeding the lowest near-ultrasound limit of $75 \mathrm{~dB}$ in the 1/3-octave band at $20 \mathrm{kHz}$ [26] at $5 \mathrm{~cm}$ from the coil for all but Magstim 70mm Double Coil and MagVenture MRi-B91 (Fig. 2). Thus, to fully capture the sound, we recommend measuring the sound to at least $30 \mathrm{kHz}$, which is much higher than the $8 \mathrm{kHz}$ or $16 \mathrm{kHz}$ bandwidth available in common sound level meters compliant with Class II or Class I of IEC 61672 (even if the meter supports measurement of peak SPL in addition to the time-averaged steady-state sound level of this standard).

The previous comparison between TMS devices used sound level meters with a slow time weighting and a measurement bandwidth of only $8 \mathrm{kHz}$ [10], which is insufficient for impulsive sounds [2]. Indeed, we obtained significantly higher sound pressure levels, and, for the three coils common in these two studies, a different order. At 100\% MSO and $25 \mathrm{~cm}$ distance in our study, the Magstim 70mm Double Coil, MagVenture Cool-B65, and NeuroStar coils registered at 108,113, and $117 \mathrm{~dB}(\mathrm{Z})$, respectively, whereas these coils produced only $90.2,78.3$, and $82.7 \mathrm{~dB}(\mathrm{~A})$, respectively, in the prior study despite the smaller measurement distance of $10 \mathrm{~cm}$ [10]. Specifically, the Magstim and MagVenture coils were respectively $18.3 \mathrm{~dB}$ and $35.1 \mathrm{~dB}$ louder with the wide-bandwidth impulse sound measurement at $25 \mathrm{~cm}$, and these numbers are estimated to rise to $26.2 \mathrm{~dB}$ and $43.1 \mathrm{~dB}$ extrapolating the loudness at a matched distance of $10 \mathrm{~cm}$. It should be noted, though, that the version of a particular coil that we tested may have differed from those in other studies; for example, the NeuroStar coil we tested is an early model (version 1.0).

On the other hand, compared to the previous peak SPL measurement using a head phantom [5], we obtained lower SPLs for the same coil (Magstim 70mm Double Coil, $108 \mathrm{~dB}(\mathrm{Z})$ in free field versus $127.6 \mathrm{~dB}(\mathrm{C})$ in the ear canal). This difference is only partially explained by the smaller distance between the ear and the coil. The main factor is likely the ear canal model itself, which mimics the fact that the pressure levels in the ear are higher than the free field pressure levels [29]. The GRAS RA0045 microphone used in those measurements attempts to mimic human ear-canal response curve between 0 and $10,000 \mathrm{~Hz}$ (and has a narrow $+32 \mathrm{~dB}$ resonant peak outside that measurement range at $13.5 \mathrm{kHz}$, likely amplifying the high-frequency content of the coil click) [28]. All mentioned $140 \mathrm{~dB}$ limits, however, are defined for free-field SPL [20-23,25]. Importantly, head phantoms 
established in acoustics do not model body sound through the skull, which depends on the stimulation location and can increase the relevant sound pressure of TMS in the ear [30].

Our measurements indicate that for an operator holding a TMS coil, the sound from single-pulse TMS and rTMS at common frequencies is below relevant exposure limits. However, the sound level can come close to the exposure limits, or even exceed them at very high pulse repetition rates. Moreover, there is emerging evidence that hearing loss is more complex than assumed in the current exposure limits [31]. So called "hidden" hearing loss, i.e., a reduction in the ability to distinguish sounds in noisy environment, has been observed already with loud noise exposure below levels causing permanent audiometric hearing loss [31]. Further, the standard limits $[20-21,23,26]$ are only a guide to maximum allowable environmental exposure, and do not guarantee safety $[21,32]$ (i.e., one should reduce exposure well below the specified limits, if possible). Finally, we characterized only a sample of figure-of-eight TMS coils; it is possible that there are or will be louder ones. Indeed, rTMS with a different kind of coil has caused permanent hearing loss [6]. Based on these considerations, we recommended that persons close to an operating TMS coil wear hearing protection.

For the TMS subject, the sound levels are even higher. Further, conventional acoustic measurements of TMS coils do not address the sound conducted through the skull, neither do they account for the possibility of significant near-field peaks of acoustic energy. Therefore, we consider hearing protection essential for TMS subjects.

\section{Conclusion}

Commonly used TMS devices have large differences in their sound levels at matched stimulation strength. The sound of the tested coils was below, but near, relevant exposure limits at distances relevant for the TMS operator. For the TMS subject, some near-ultrasound limits may be exceeded and there may be other sources of risk such as skull conduction and near-field sound pressure peaks. Generally, sound exposure standards may inadequately capture some risks to hearing. Therefore, we recommend hearing protection for persons near operating TMS coils, and we consider hearing protection essential for the TMS subject.

\section{Conflict of Interest}

L. M. Koponen, S. M. Goetz, and A. V. Peterchev are inventors on patents and patent applications on TMS technology including TMS devices with reduced acoustic noise. S. M. Goetz has received research funding from Magstim Inc. A. V. Peterchev has received research and travel support as well as patent royalties from Rogue Research; research and travel support, consulting fees, as well as equipment donation from Tal Medical / Neurex; patent application and research support from Magstim; as well as equipment loans from MagVenture, all related to TMS technology.

\section{Acknowledgements}

Research reported in this publication was supported by the National Institute of Mental Health of the National Institutes of Health under Award Number R01MH111865. The content is solely the responsibility of the authors and does not necessarily represent the official views of the National Institutes of Health.

\section{References}

1. Counter SA, Borg E. Analysis of the coil generated impulse noise in extracranial magnetic stimulation. Electroencephalogr Clin Neurophysiol 1992;85:280-8. 
2. Goetz SM, Lisanby SH, Murphy DL, Price RJ, O'Grady G, Peterchev AV. Impulse noise of transcranial magnetic stimulation: measurement, safety, and auditory neuromodulation. Brain Stimul 2015;8:1613.

3. Pascual-Leone A, Cohen LG, Shotland LI, Dang N, Pikus A, Wassermann EM, et al. No evidence of hearing loss in humans due to transcranial magnetic stimulation. Neurology. 1992;42:647-51.

4. Tringali S, Perrot $X$, Collet L, Moulin A. Repetitive transcranial magnetic stimulation: Hearing safety considerations. Brain Stimul 2012;5:354-63.

5. Kukke SN, Brewer CC, Zalewski C, King KA, Damiano D, Alter KE, et al. Hearing Safety from Single-and Double-Pulse transcranial magnetic stimulation in children and young adults. J Clin Neurophysiol 2017;4:340-7.

6. Zangen $A$, Roth $\mathrm{Y}$, Voller $\mathrm{B}$, Hallett $\mathrm{M}$. Transcranial magnetic stimulation of deep brain regions: evidence for efficacy of the H-coil. Clin Neurophysiol 2005;116:775-9.

7. Nikouline V, Ruohonen J, Ilmoniemi RJ. The role of the coil click in TMS assessed with simultaneous EEG. Clin Neurophysiol 1999;110:1325-8.

8. Ter Braack EM, de Vos CC, van Putten MJ. Masking the auditory evoked potential in TMS-EEG: a comparison of various methods. Brain Topography 2015;28:520-8.

9. Rossi S, Hallett M, Rossini PM, Pascual-Leone A, Safety of TMS Consensus Group. Safety, ethical considerations, and application guidelines for the use of transcranial magnetic stimulation in clinical practice and research. Clin Neurophysiol. 2009;120:2008-39.

10. Dhamne SC, Kothare RS, Yu C, Hsieh TH, Anastasio EM, Oberman L, et al. A measure of acoustic noise generated from transcranial magnetic stimulation coils. Brain Stimul 2014;7:432-4.

11. ANSI/ASA S1.4-2014/Part 1. American National Standard Electroacoustics - Sound Level Meters - Part 1: Specifications (a nationally adopted international standard). Acoustical Society of America, Melville, NY 11747; 2014.

12. Huang YZ, Edwards MJ, Rounis E, Bhatia KP, Rothwell JC. Theta burst stimulation of the human motor cortex. Neuron 2005;45:201-6.

13. Deng ZD, Lisanby SH, Peterchev AV. Electric field depth-focality tradeoff in transcranial magnetic stimulation: simulation comparison of 50 coil designs. Brain Stim 2013;6:1-13.

14. Ilmoniemi RJ. The triangle phantom in magnetoencephalography. J Jpn Biomagn Bioelectromagn Soc 2009;22:44-5.

15. Nieminen JO, Koponen LM, Ilmoniemi RJ. Experimental characterization of the electric field distribution induced by TMS devices. Brain Stim 2015; 8:582-9.

16. Barker AT, Garnham CW, Freeston IL Magnetic nerve stimulation: The effect of waveform on efficiency, determination of neural membrane time constants and the measurement of stimulator output. Electroencephalogr Clin Neurophysiol Suppl 1991;43: 227-37.

17. Peterchev AV, Goetz SM, Westin GG, Luber B, Lisanby SH. Pulse width dependence of motor threshold and input-output curve characterized with controllable pulse parameter transcranial magnetic stimulation. Clin Neurophysiol 2013; 124:1364-72.

18. Goetz SM, Murphy DL, Peterchev AV. Transcranial magnetic stimulation device with reduced acoustic noise. IEEE Mag Lett 2014;5:1500104.

19. Kammer T, Beck S, Thielscher A, Laubis-Herrmann U, Topka, H. Motor thresholds in humans: A transcranial magnetic stimulation study comparing different pulse waveforms, current directions and stimulator types. Clin Neurophysiol 2001;112:250-8. 
20. Occupational Safety and Health Admin, Occupational noise exposure, 29 CFR 1910.95(b)(1), https://www.govinfo.gov/content/pkg/CFR-2010-title29-vol5/pdf/CFR-2010-title29-vol5-sec191095.pdf; [accessed 25 March 2019]

21. Occupational Safety and Health Admin, Occupational noise exposure, 29 CFR 1926.52I, https://www.govinfo.gov/content/pkg/CFR-2014-title29-vol8/pdf/CFR-2014-title29-vol8-sec192652.pdf; [accessed 25 March 2019]

22. Occupational Safety and Health Admin, Technical manual, https://www.osha.gov/dts/osta/otm/new_noise/; 2013 [accessed 25 March 2019]

23. American Conference of Governmental Industrial Hygienists. TVLs and BEls. Cincinnati: ACGIH; 2012.

24. Peterchev AV, Murphy DL, Goetz SM. Quiet transcranial magnetic stimulation: Status and future directions. 37th Annual International Conference of the IEEE Engineering in Medicine and Biology Society (EMBC) 2015:226-9.

25. MIL-STD-1474E. Department of Defense design criteria standard noise limits, AMSC 9542, Washington, DC; 2015.

26. Duck FA. Medical and non-medical protection standards for ultrasound and infrasound. Progress Biophys Molecular Biol 2007;93:176-91.

27. Starck J, Rimpiläinen I, Pyykkö I, Esko T. The noise level in magnetic stimulation. Scandinavian Audiology 1996;25:223-6.

28. Shaw EAG. Transformation of sound pressure level from the free field to the eardrum in the horizontal plane. J Acoustical Soc America 1974;56:1848-61.

29. GRAS. GRAS RA0045 externally polarized ear simulator according to IEC 60318-4 (60711), https://www.gras.dk/products/ear-simulator/product/248-ra0045; 2019 [accessed 18 March 2019].

30. Stenfelt S, Goode RL. Bone-conducted sound: physiological and clinical aspects. Otology \& Neurotology 2005;26:1245-61.

31. Plack CJ, Barker D, Prendergast G. Perceptual consequences of "hidden" hearing loss. Trends Hear 2014;18: 2331216514550621.

32. Fink DJ. What is a safe noise level for the public? Am J Public Health 2017;107:44-5. 


\section{Tables and figures}

Table 1. Tested TMS stimulators and coils from Magstim (UK), MagVenture (Denmark), and Neuronetics (USA).

\begin{tabular}{l|l} 
Stimulator & Coil \\
\hline Magstim Rapid2 & 70mm Double Coil (P/N 9925-00) \\
\cline { 2 - 2 } (P/N 3013-US) & AirFilm Coil (P/N 3910-00) \\
\hline \multirow{3}{*}{$\begin{array}{l}\text { MagVenture MagPro X100 incl. MagOption } \\
\text { (P/N 9016E0731) }\end{array}$} & C-B60 (P/N 9016E0482) \\
\cline { 2 - 2 } & Cool-B65 (P/N 9016E0491) \\
\cline { 2 - 2 } $\begin{array}{l}\text { Neuronetics NeuroStar TMS Therapy System } \\
\text { (ver. 1.0, P/N 81-00315-000) }\end{array}$ & MC-B70 (P/N 9016E0564) \\
\cline { 2 - 2 } & MRi-B91 (P/N 9016E0661) \\
\hline
\end{tabular}

${ }^{*}$ Connected to stimulator with adapter (Magstim, P/N 3110-00).

** "Standard biphasic" pulse mode was used.

Table 2. Scaling laws for reported peak SPL and rTMS sound level.

\begin{tabular}{|c|c|c|}
\hline Effect & Change in peak SPL & Change in rTMS sound level \\
\hline \multirow{2}{*}{$\begin{array}{l}\text { Stimulation } \\
\text { strength }\end{array}$} & Stim. strength [\% RMT] & Stim. strength [\% RMT $]$ \\
\hline & $12 \mathrm{~dB} \cdot \log _{2}$ & $12 \mathrm{~dB} \cdot \log _{2}$ \\
\hline \multirow{2}{*}{ Repetition rate } & \multirow{2}{*}{0} & \multirow{2}{*}{$3 \mathrm{~dB} \cdot \log _{2} \frac{\text { Rate }[\mathrm{Hz}]}{15 \mathrm{~Hz}}$} \\
\hline & & \\
\hline $\begin{array}{l}\text { Distance from } \\
\text { coil }^{*}\end{array}$ & $-6 \mathrm{~dB} \cdot \log _{2} \frac{\text { Distance }[\mathrm{cm}]}{25 \mathrm{~cm}}$ & $-6 \mathrm{~dB} \cdot \log _{2} \frac{\text { Distance }[\mathrm{cm}]}{25 \mathrm{~cm}}$ \\
\hline
\end{tabular}

${ }^{*}$ Valid down to distance of $5 \mathrm{~cm}$ [27]. 

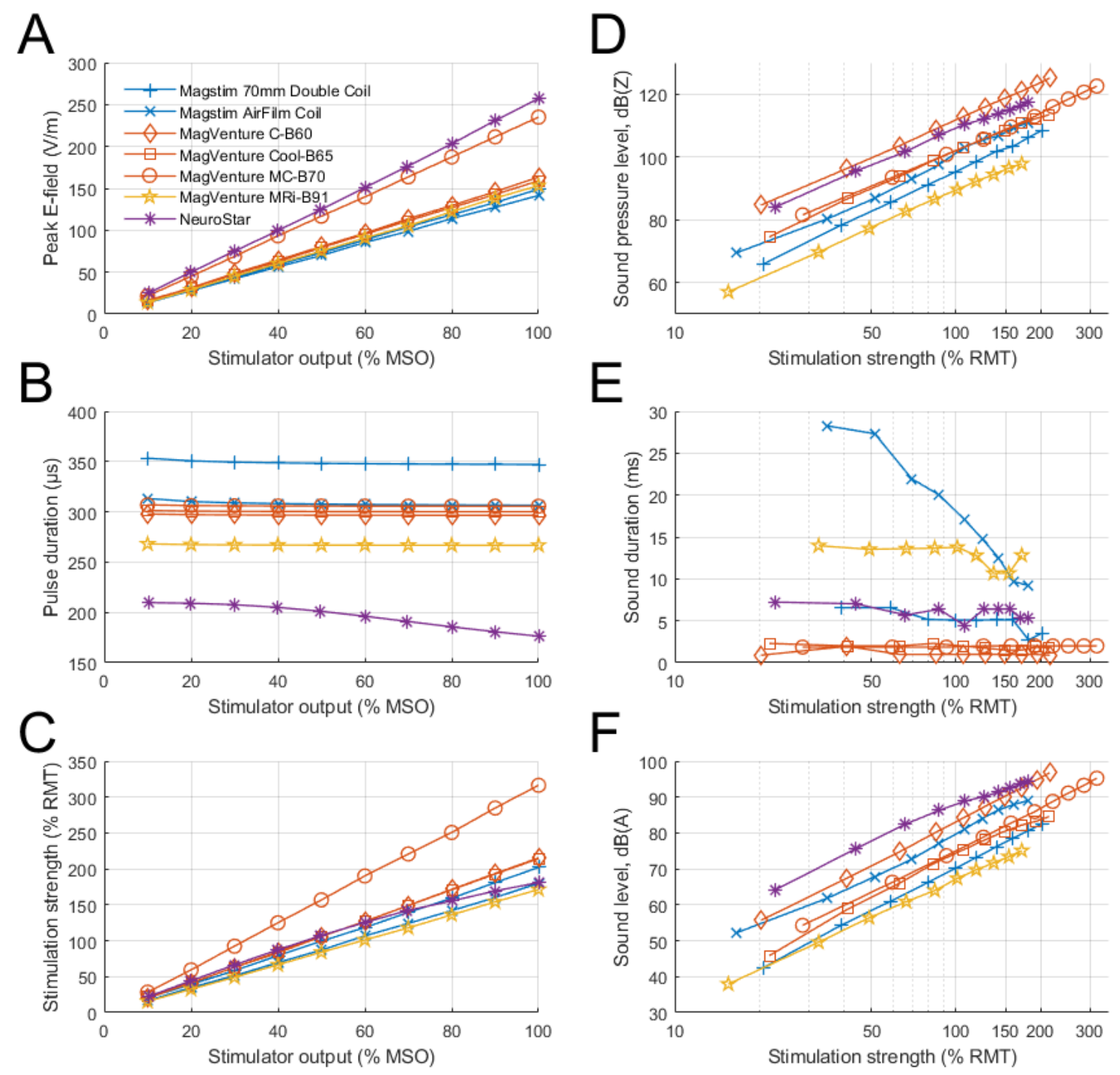

Figure 1. E-field and sound characteristics of various measured TMS coils. (A) Peak E-field value has approximately linear relationship with stimulator output. (B) Pulse durations of air-core coils are approximately constant, whereas pulse duration of NeuroStar iron-core coil starts to drop after about $30 \% \mathrm{MSO}$. (C) Stimulation strength estimated with neural model, where $50.3 \%$ MSO of Magstim 70mm Double Coil is set to correspond to $100 \%$ RMT. (D) Peak sound pressure level (SPL) with Z-weighting, i.e., flat response curve. (E) Duration for which sound is within $20 \mathrm{~dB}$ of peak value. (F) Simulated sound level of a $15 \mathrm{~Hz}$ rTMS pulse train with A-weighting (slow time weighting). 

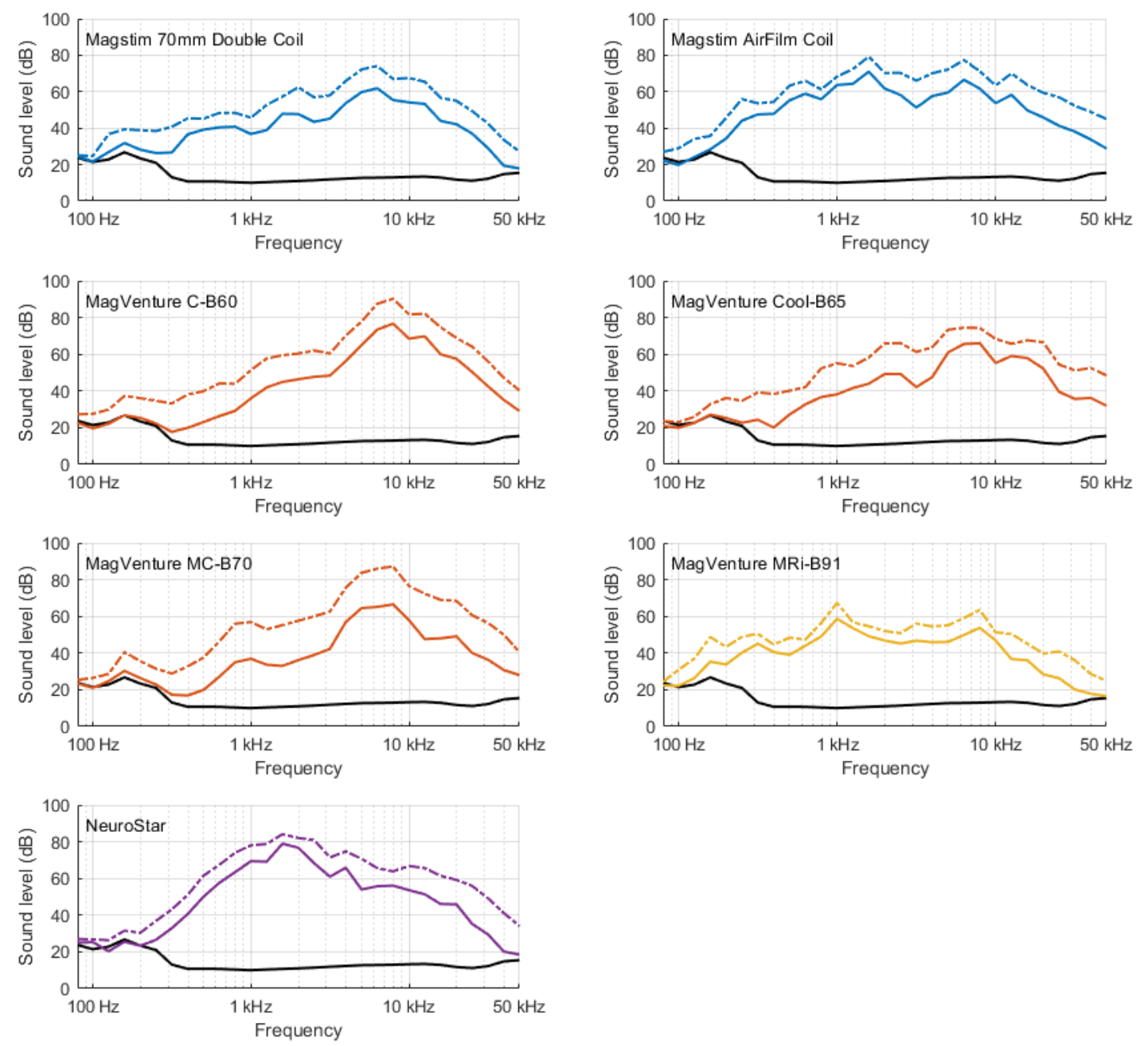

Figure 2. 1/3-octave spectra of tested coils at estimated 100\% RMT (solid line) and at $100 \%$ MSO (dash-dotted line). Solid black line shows ambient sound spectrum. For all coils, spectra power peak is between 1,000 and $10,000 \mathrm{~Hz}$, i.e., within hearing range $(20-20,000 \mathrm{~Hz})$. Spectra at $100 \%$ RMT were produced from closest measured stimulator output by assuming that sound waveform is proportional to pulse energy. Due to the $0.2 \mathrm{~s}$ time window for each sample, the sound level corresponds to that of a $5 \mathrm{~Hz}$ rTMS pulse train. 


\section{Supplementary methods}

\section{Sound recordings}

Based on preliminary measurements, the impulsive sound from TMS pulse generators can be relatively loud. In preliminary measurements with the Tonica MagPro MST stimulator (MagVenture, Denmark), using the same 25 and $100 \mathrm{~cm}$ distances from the coil to the microphones as in the final measurement dataset, an impulsive sound source with a peak SPL of $104 \mathrm{~dB}(\mathrm{Z})$ at 1.7 meters away from the farther microphone was identified. This distance corresponded with the distance to the stimulator. This sound was just $9 \mathrm{~dB}$ less than the sound from the tested coil at $1.0 \mathrm{~m}$, i.e., only about $6 \mathrm{~dB}$ less if both sound sources would be at equal distances.

To measure accurately the waveform of the coil sound, we had to isolate it from the impulsive sound of the stimulator. We achieved this isolation by measuring the coils inside a soundproof chamber with the TMS pulse generator outside. If one is interested only in the peak SPL and measures from a reasonably close distance to the coil, a soundproof chamber will not be needed. However, in such case, one might want to use at least two microphones (and beamforming) to identify and, if needed, to reject the sound not originating from the direction of the coil. The supplementary sound recording dataset contains a second microphone at $100 \mathrm{~cm}$ from the coil, but due to the soundproof chamber the data from this microphone was not needed in the analysis. For the placement of the microphones and the acoustic foam, see Fig. S1B.

The peak SPL was defined as

$$
\mathrm{SPL}=\max _{t \in T}\left(94+20 \log _{10} \frac{|p(t)|}{1 \mathrm{~Pa}}\right) \mathrm{dB}(\mathrm{Z})
$$

where $T$ is the $0.2 \mathrm{~s}$ time window around a trigger event (from $-50 \mathrm{~ms}$ to $150 \mathrm{~ms}$ ) and $p(t)$ is the filtered instantaneous sound pressure [S1]. The ambient noise measured in the soundproof chamber had SPL of $45.0 \mathrm{~dB}(\mathrm{Z})$ (95\% confidence interval of 43.3-46.6 dB(Z)) with Z-weighting and sound level of $21.2 \mathrm{~dB}(\mathrm{~A})$ with Aweighting. Consequently, we could measure the pulse durations for pulses down to about $70 \mathrm{~dB}(\mathrm{Z})$, i.e., down to $20 \%$ MSO for the three quietest coils and down to $10 \%$ MSO for the other coils.

As a supplementary material we provide the full set of sound recordings, band-pass filtered from 60 to $60,000 \mathrm{~Hz}$, as described in the methods, and compressed to contain data from $-50 \mathrm{~ms}$ to $150 \mathrm{~ms}$ relative to each pulse, in Matlab file "sound.mat". In this file, the originally 24-bit sound recording data were converted to Pascals and stored as 32-bit floating point numbers. In addition to the coil sounds, to assess the background noise level, there are also recordings of 100 events in which no pulse was delivered. 


\section{Electric field measurements}

The coil E-field was measured with a 70-millimeter-high printed-circuit-board triangular pickup loop with a 50ohm series resistor, a 3d-printed holder, and a laser-cut alignment tool for mounting the holder on the coils (Fig. S1A). The E-field measurements were estimated to have $<2 \mathrm{~mm}$ positional $(<0.05 \mathrm{~mm}$ for distance from the bottom of the coil) and $<2^{\circ}$ angular uncertainty. With these uncertainties, the field values for each coil have $<2 \%$ gain uncertainty. The technical drawings of the measurement probe are provided in file "probe.zip" containing the Gerber files for the two-layer printed-circuit-board probe, an STL file for the 3d-printed probe holder, and a SVG drawing of the laser-cut alignment plate.

The full E-field measurement dataset, including the pulse waveforms, with one pulse per coil per stimulator output, sampled at $100 \mathrm{MHz}$, is provided in Matlab file "stimulation.mat".

The stimulation strength was computed from the E-field recordings as

$$
\Delta V=\max _{t}\left|\int_{0}^{t} E\left(t^{\prime}\right) \exp \left(-\frac{t-t^{\prime}}{\tau}\right) \mathrm{d} t^{\prime}\right|,
$$

where $\Delta V$ is the stimulation strength, $E(t)$ is the measured E-field waveform, and $\tau$ is the strength-duration time constant, here $200 \mu \mathrm{s}$.

\section{References}

S1. ANSI/ASA S1.4-2014/Part 1. American National Standard Electroacoustics - Sound Level Meters - Part 1: Specifications (a nationally adopted international standard). Acoustical Society of America, Melville, NY 11747; 2014. 


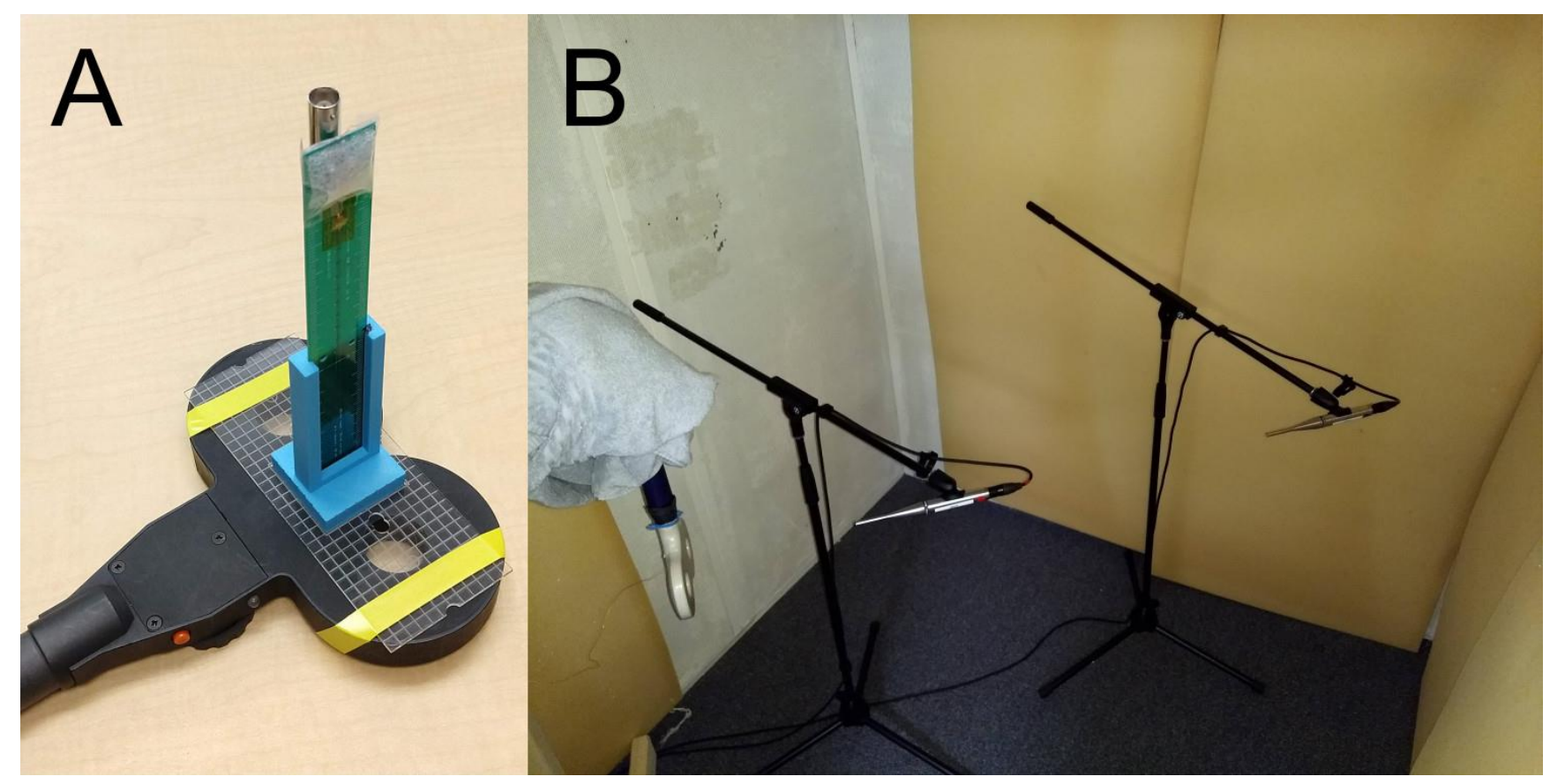

Figure S1. (A) Triangular E-field probe in 3d-printed holder on laser-cut alignment plate positioned on example TMS coil. (B) Sound-recording setup: two microphones are directed at head-side of coil at respective distance of $25 \mathrm{~cm}$ and $100 \mathrm{~cm}$ measured from microphone capsule. Soundproof chamber provided adequate attenuation of external sound sources including TMS pulse generator; therefore, only data from closer microphone was ultimately needed for analysis. Corners behind coil and microphones were covered with $50 \mathrm{~mm}$ thick open-cell foam to suppress echoes. Coil cable is wrapped in towels to suppress its sound.

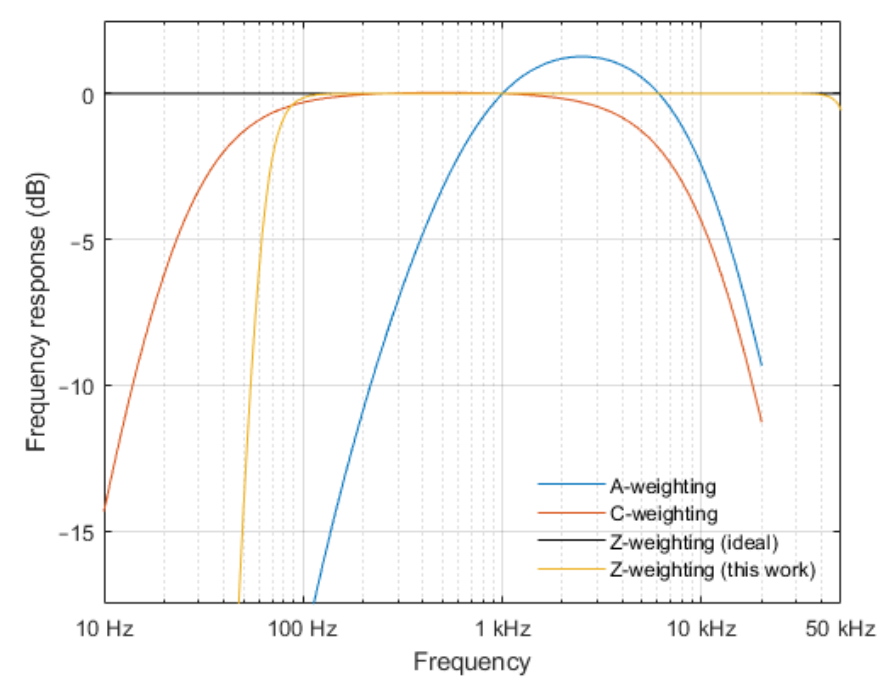

Figure S2. Frequency weightings used in hearing safety standards. A-weighting roughly mimics perceived loudness curve for barely audible sounds, whereas C-weighting mimics that of loud sounds. Ideal Z-weighting corresponds to no weighting at all. In this work, Z-weighting filter had passband from 80 to $50,000 \mathrm{~Hz}$, with sharp low-frequency cut-off selected to reduce noise from non-TMS-coil sources. In practice, a Class II soundlevel meter may omit anything above $8 \mathrm{kHz}$, and a Class I sound-level meter anything above $16 \mathrm{kHz}$. 